\title{
Neuroprotective Effects of Licorice Extracts in Tibial and Sural Transection Induced Neuropathic Pain in Rats
}

\author{
Tanmayee Amit Joshi', Pratima Tatke ${ }^{2, *}$ \\ 1Department of Pharmacology C. U. Shah College of Pharmacy, SNDT Women's University, Sir Vithaldas Vidyavihar, \\ Juhu Campus, Santacruz (W), Mumbai, Maharashtra, INDIA. \\ ${ }^{2}$ Department of Pharmaceutical Chemistry C. U. Shah College of Pharmacy, SNDT Women's University, Juhu Campus, \\ Santacruz (W), Mumbai, Maharashtra, INDIA.
}

\begin{abstract}
Background: Neuropathic pain (NP) is the worst of tortures, a nerve wound may experience as a result of nerve damage or complication of diabetes/HIV/cancer. There are no defined guidelines for treatment. Available treatments have various side effects. Glycyrrhiza glabra (Licorice) has not been explored scientifically with respect to NP. Hence there is a dire need to develop treatment for NP which will be safe, effective and can be taken for prolonged time. The present study is to evaluate effects of licorice extracts in Tibial and Sural Transection (TST) induced neuropathic pain in rats. Materials and Methods: Extracts prepared from crude drug were tested for antioxidant activity. NP was induced by TST surgery method. After induction of NP, it leads to chemical and heat evoked hyperalgesia, cold and mechanical allodynia. Rats were divided into eight groups, Control (Treated with $\mathrm{Na}-\mathrm{CMC}$ ), Standard (Treated with Pregabalin), Test (Treated with three licorice extracts), Sham operated and Normal (Without surgery). Rats were treated with extracts and standard for 21 days. At the end of 21 days' rats were sacrificed and tested for biochemical parameters. At the end of study biochemical parameters; superoxide generation, malondialdehyde (MDA) and TNF- $\alpha$ were evaluated. Results: Rats treated with licorice extracts showed improvement all the pain related symptoms and tibial functional index. Treated rats also showed significant improvement in superoxide ions and MDA supporting their antinociceptive and antioxidant activities. Conclusion: Licorice extracts have shown significant decrease in pain symptoms in TST induced NP in rats. This effect may be attributed to anti-inflammatory, antioxidant and neuroprotective properties of licorice. Hence licorice extracts can be potential candidates for treatment of NP.

Key words: Glycyrizza glabra, Neuropathic pain, Tibial and Sural Transection, Antioxidant, Licorice root, Antiinflammatory.
\end{abstract}

\section{INTRODUCTION}

Pain is a troublesome sensory and emotional encounter associated with actual or potential tissue damage. ${ }^{1}$ Pain that lasts a long time is called chronic pain. Neuropathic pain is a type of chronic pain which can occur due to diseases or damage to somatosensory nervous system. Neuropathic pain is prominently experienced as a complication of diabetes, Herpes- zoster infection, HIV infection and cancer. ${ }^{2}$ Neuropathic pain is an area of huge medical need as available therapies are inadequate and nonspecific. The commonly used drugs for treatment of neuropathic pain include gabapentin, pregabaline, amitryptiline, desipramine, opioid analgesics etc. The current treatment has many side effects, such as blurred vision, tachycardia, orthostatic hypotension, GIT disorders, asthenia, peripheral edema etc. Hence, there is a need of new, safe and effective treatment strategies. ${ }^{3,4}$ The pain interferes with sleep, enjoyment, mobility;
Submission Date: 29-09-2019; Revision Date: 26-12-2019; Accepted Date: 12-05-2020

DOI: 10.5530/ijper.54.2s.85 Correspondence: Dr. Pratima Tatke Principal and Professor of Pharmaceutical Chemistry, C. U. Shah College of Pharmacy, SNDT Women's University, Juhu Campus, Santacruz (W), Mumbai, Maharashtra, INDIA.

Phone: +919920685857 E-mail: drpratimatatke@ gmail.com

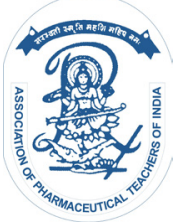

www.ijper.org 
hence decreasing quality of life of patients. ${ }^{5}$ Neuropathic pain also exists concomitantly with other diseases, patients also suffer from adverse effects from their respective primary disease and another treatment for neuropathic pain increases burden of adverse effects too. Plants are available as reliable alternative as herbal therapies are devoid of adverse effects and they can be consumed for prolonged period. ${ }^{6}$

Various preclinical models for inducing neuropathic pain include; spinal nerve ligation, chronic constriction injury, spared nerve injury and Tibial and Sural transection. ${ }^{7}$ Although chronic constriction injury is commonest used model, it has certain disadvantages. The tension induced by the ligatures around the nerve can easily vary, the degree of constriction may vary from one experimenter to other and number of sciatic nerve axons ligated each time is difficult to reproduce. Tibial and Sural Transection (TST) model by Lee $e t a l$. produces most pronounced pain syndrome, with development of symptoms like allodynia and hyperalgesia. ${ }^{8}$

The pathophysiology of neuropathic pain is complex. The major pathophysiological factors included in genesis of neuropathic pain are, ectopic firing from sodium ion channels, presence of inflammatory mediators and overproduction of Reactive Oxygen Species (ROS). Ectopic action potential created from sodium channels leads to symptoms of spontaneous pain whereas excessive inflammation and ROS can result in hyperalgesia and allodynia, which are symptoms of stimulus dependent pain. ${ }^{9,10}$ In chronic constriction injury (CCI) model of rat neuropathic pain, heat hyperalgesia was reportedly attenuated by systemically administered antioxidants. ${ }^{11,12}$ Hence the medicinal agent with antioxidant and anti-inflammatory properties can be potential candidate, to investigate its effect in neuropathic pain.

The species Glycyrrbiza glabra L. (Common name: Licorice) has well known therapeutic properties which have been known since ages. Licorice contains number of compounds such as triterpenesaponin, flavonoids, polysaccharides, asparagine, bitters, estrogen, gums, mucilage, protein, resins, starches, sterols, volatile oils, tannins, glycosides and various other substances. This plant is used traditionally in the treatment of cough and ulcers. As a part of folklore medicine, it is also used as laxative, contraceptive, antiviral agent and galactogogue. Licorice is also reported to have activities like hepatoprotective, antibacterial, antiviral, antidiabetic, anti-tumor, immunomudulator etc. ${ }^{13}$ The constituents of licorice also exhibit steroid-like anti-inflammatory activity, similar to the action of hydrocortisone. This anti-inflammatory activity is due to inhibition of phospolipase $A_{2}$, an enzyme which is essential for numerous inflammatory processes. The plant also exhibits significant anti-oxidant activity. ${ }^{14}$ Glycyrrhetinic acid, a triterpenoid saponin obtained from licorice has been proven to be an anti-inflammatory agent. Literature search also reveals anti-nociceptive activity from other saponin containing medicinal plants such as Acorus calamus ${ }^{15}$ and Ocimum sanctum. ${ }^{16}$ $18 \beta$-Glycyrrhetinic acid which is a well- known constituent of licorice, has also shown antioxidant effects in mice model by scavenging superoxide radicals. ${ }^{17}$ The present study was designed to investigate neuroprotective properties of licorice extracts for TST induced neuropathic pain.

\section{MATERIALS AND METHODS}

\section{Animal Studies}

Wistar albino rats (weight range of 180 to $250 \mathrm{gm}$ ) were procured from Bharat Serums and Vaccines Ltd. Thane, India. The animals were kept in the polycarbonate animal cages with access to purified water and standard laboratory pellet chow diet ad libitum. (Pranav Agro Ltd., Pune, India). The rats were exposed to a $12: 12 \mathrm{hr}$ light: dark cycle. The protocol for pre-clinical experiment was approved by the Institutional Animal Ethics Committee (IAEC) and the animals were looked after as per the guidelines by of the CPCSEA, under the expert supervision of a veterinarian. (Reg.No.- 39/PO/ $\mathrm{Re} / \mathrm{S} /$ 99/CPCSEA)).

\section{Drugs and reagents}

The dried roots and rhizomes of licorice were obtained from Shreeji Herbals. The parts of the plants were further authenticated from Agharkar Institute Pune, with letter number No.3/187/2015/Adm-2796 with authentication number, 15-175. Pregabalin was gifted by Alembic Pharma. Xanthine, NBT (Nitro Blue Tetrazolium), 2, 2'-diphenyl-1- picrylhydrazyl (DPPH•) was procured from Himedia, Bovine Brain Extract (BBE), MDA, Xanthine oxidase were procured from Sigma for the present study. TNF- $\alpha$ kit was purchased from Bioassay laboratory technology, China. The analytical grade chemicals were used in the current study.

\section{Extraction}

The dried roots were procured and were powdered in a mixer. Powdered licorice was extracted with respective solvents in the drug: solvent ratio of 1:5 using soxhlet extraction method. The extraction was carried out for $30 \mathrm{hr}$. The extracts were filtered and dried. The extracts were stored in amber colored glass bottle in dessicator 
and used for further study. The yield and properties of the extracts are as given below:

\section{Estimation of total phenolic content and total flavonoid content}

Phenolic compounds as well as flavonoids are natural antioxidants. Hence all the prepared extracts and formulation were screened initially by determining Total Phenolic Content (TPC) of the prepared extracts by means of the Folin-Ciocalteu phenol reagent. ${ }^{18}$ Total flavonoid content was calculated by the aluminum chloride colorimetric assay. ${ }^{19}$ Extracts with good TPC and flavonoid content were further tested for antioxidant activity.

Antioxidant activity of the extracts was carried out by three different methods as follows

\section{DPPH scavenging activity of extracts}

The antioxidant activity is expressed in terms of radical scavenging capacity in reaction with a stable radical known as 2, 2'-diphenyl-1- picrylhydrazyl (DPPH•) The extracts were reacted with react with stable DPPH radical. The decrease in absorbance $\mathrm{DPPH} \bullet$ of at a particular wavelength was measured.

$1 \mathrm{ml}$ of the extract solution and $3 \mathrm{ml}$ of $0.1 \mathrm{mM}$ solution of DPPH dissolved in methanol was allowed to react. The solution was kept in dark for $30 \mathrm{~min}$. Absorbance was measured at $517 \mathrm{~nm}$ using UV visible spectrophotometer. The percentage scavenging activity was calculated by using the standard formula of absorbance. The $\mathrm{IC}_{50}$ values scavenging $50 \%$ of DPPH radical were estimated. ${ }^{20}$

\section{Lipid peroxidation products scavenging activity of extracts}

BBE Bovine Brain Extract (BBE) was dissolved in phosphate buffer saline (PBS) and was sonicated in ice bath. BBE was preincubated with plant extracts along with $\mathrm{PBS}$ and $\mathrm{FeCl}_{3}$. The reaction of lipid peroxidation was triggered by addition of ascorbate and the mixture was incubated. The reaction was halted by adding trichloroacetic acid and 2-thiobarbituric acid (TBA). The resultant mixture was heated at $100^{\circ} \mathrm{C}$ for $1 \mathrm{hr}$ in a water bath. The chromogen complex formed was measured at $532 \mathrm{~nm}$ after its extraction into 1-butanol spectrophotometrically. . The $\mathrm{IC}_{50}$ values of the extracts are determined. ${ }^{21}$

\section{Superoxide anion scavenging method}

A reaction mixture consisting of disodium EDTA (prepared in buffer of $\mathrm{KH}_{2} \mathrm{PO}_{4} / \mathrm{KOH}, \mathrm{pH} 7.4$ ), NBT chloride and xanthine was prepared and extracts were incubated at room temperature in this mixture. The reaction to form superoxide was initiated by addition of xanthine oxidase solution. The change in absorbance of NBT due to its reduction by superoxides was measured at $560 \mathrm{~nm}$ using the microplate reader. ${ }^{22}$

\section{Induction of neuropathic pain by tibial and sural transection}

The neuropathic pain was induced by TST method as described by Lee $e t a .^{\$}$ The rats were anesthetesized by ketamine $(75 \mathrm{mg} / \mathrm{kg})$ followed by xylazine $(5 \mathrm{mg} / \mathrm{kg})$. The left hind limb was shaved off. The hind limb was fixed to the surface with the help of cello tape. A cut was given through the biceps femoris muscle to show the sciatic nerve, which lies just below the biceps femoris. The three terminal branches i.e. the sural, common peroneal and tibial nerves were exposed too. Then distal to the trifurcation, $2 \mathrm{~mm}$ nerve sections of tibial and sural were ligated and cut. The common peroneal nerve was not ligated. The layers of muscle and skin were closed and stitched. The animals in sham control group were left without any ligations, the sciatic nerve and its three branches were only exposed. ${ }^{23}$ All the procedures were performed by same experimenter, in sterile conditions under the supervision of veterinarian. The animals were divided into groups as follows: Normal rats (No surgery), Control (TST $+\mathrm{NaCMC}$ ), sham (sham operated $+\mathrm{Na} \mathrm{CMC}$ ), treatment with pregabalin $(10 \mathrm{mg} / \mathrm{kg})$, standard drug (TST + pregabalin), treatment with methanol extract $200 \mathrm{mg} / \mathrm{kg}$ (TST+ LME), treatment with ethanol extract $200 \mathrm{mg} / \mathrm{kg}$ $($ TST + LE) and treatment with ethyl acetate extract $200 \mathrm{mg} / \mathrm{kg}$ (TST + LEA). The extracts were administered orally for period of 21 days.

\section{Behavioral examination}

Identifying intensity of neuropathic pain in clinical as well as preclinical studies is not always straightforward. A standardized bedside examination of patients with neuropathic pain includes various tests for sensation of touch, pin prick, pressure, cold, heat and vibration. Different models of neuropathies (surgery induced, chemically-induced, diabetes-associated) have been successfully established in the rat and different procedure to record their response to nociceptive stimulations are well reported in the literature. ${ }^{24}$ Hence the behavioral examinations done at preclinical level correlate to the pain assessment at clinical level.

\section{Heat hyperalgesia (Eddy's Hot Plate)}

Thermal hyperalgesia, can be evaluated by measuring by thermal nociceptive threshold. It can be measured by the Eddy's hot plate also known as (analgesiometer). It is a well-known method described by Eddy and 
co-workers to measure thermal sensitivity. During the procedure, the plate was heated and temperature of $51.5 \pm 0.5^{\circ} \mathrm{C}$ was maintained. The rat was positioned on the hot plate and nociceptive threshold was recorded with regards to licking of the paws and/or jumping in seconds. The cut-off time of $15 \mathrm{~s}$ was maintained. ${ }^{25}$

\section{Mechanical hyperalgesia (Pin prick test)}

Pinprick test as reported by Erichsen and BlackburnMunro was used to evaluate mechanical hyperalgesia. The operated hind paw was pricked with the point of an 18 gauge needle. Pricking was done with sufficient force to cause a reflex withdrawal response in nonoperated animals. However, care was taken not to penetrate the skin. The duration of the paw withdrawal was measured by a stopwatch in seconds. A cut-off time of $20 \mathrm{~s}$ was maintained. ${ }^{26}$

\section{Cold chemical allodynia (Acetone drop method)}

Acetone drop method reported by Choi et al. Is simple method to assess reactivity of animals to non-injurious cold stimulus. During the test, the rat was put on the top of a wire mesh grid. $100 \mu \mathrm{l}$ acetone was sprayed on the operated surface of paw of rat with the help of spray bottle. Paw lifting reaction was considered as reaction to cold stimuli and was recorded. ${ }^{27}$

\section{Mechanical dynamic allodynia (Paint Brush Method)}

Mechanical stimulus was evaluated by the "paint-brush" behavioural test, to investigate dynamic responses of a rat. ${ }^{28}$ This method is used for measurement for allodynia as rubbing of smooth paint brush is considered as non-noxious stimuli. Rat was positioned on the wire mesh floor. A smooth paint-brush was rubbed on the plantar area of operated hind paw. The paint brush was applied from the heel to the toes. This stimulus was applied for five times with a time interval of $5 \mathrm{sec}$ and paw lifting duration in seconds was measured. ${ }^{29}$

\section{Tibial function index}

Study of the footprints can be effectively used to estimate neurological defects in experimental rats. Medinaceli et al. has described a method for measuring the footprints of walking rats. This method consists of a walking trial of rats. The hind paws of rats were dipped in blue ink and rats were made to walk on a white paper in a corridor measuring $8.0 \mathrm{~cm} \times 62.0 \mathrm{~cm}$. Various measurements were recorded from the obtained footprints and the Tibial Function Index (TFI) was calculated by the equation given below. TFI of zero was considered as normal function of the tibial nerve, whereas a TFI of $-100 \%$ was considered to denote complete functional loss. This method is easy and dependable. It provides a measure for assessing the functional condition of the sciatic nerve. ${ }^{30,31}$

$$
\begin{gathered}
\text { TFI }=-37.2[(\mathrm{EPL}-\mathrm{NPL}) / \mathrm{NPL}]+104.4 \\
{[(\mathrm{ETS}-\mathrm{NTS}) / \mathrm{NTS}]+45.6[(\mathrm{EIT}-\mathrm{NIT}) / \mathrm{NIT}]-8.8}
\end{gathered}
$$

In the above formula, Print Length (PL) is the distance between the heel and top of the third toe; Toe Spread (TS) is distance from the first to the fifth toe and Intermediary Toe Spread (IT) denotes distance from the second to the fourth toe. The above mentioned measurements were taken both from the non-operated foot. E: Experimental and N: Non-Experimental

\section{Biochemical Estimations}

All the experimental rats were sacrificed on day 21 after surgery with carbon dioxide inhalation. The distal portions of the sciatic nerve and the tissue below the sciatic nerve were separated immediately. The samples were stored at $-20^{\circ} \mathrm{C}$. The sciatic nerve homogenate $(10 \%, \mathrm{w} / \mathrm{v})$ was prepared in $0.1 \mathrm{M}$ Tris- $\mathrm{HCl}$ buffer (pH 7.4). This homogenate was used for measurement of total protein content and thiobarbituric acid reactive substances. Superoxide anion measurement was performed on sciatic nerve. TNF- $\alpha$ determination from sciatic nerve homogenate was also performed.

\section{Estimation of superoxide anion generation}

Wang et al. has described the method to estimate the amount of superoxide anion production from tissue homogenates, by calculating reduced NBT. During the procedure, sciatic nerve homogenate was reacted with NBT to form formazan. Generated formazan is a measure of superoxide anion generation. The absorbance of formazan was assessed at $540 \mathrm{~nm}$ by UV-Vis spectrophotometer. The quantity of NBT reduction was calculated by the formula given by Wang. et al. Results were presented as picomoles per minute per milligram wet weight of sciatic nerve. ${ }^{32}$

\section{Malondialdehyde content}

The method reported by Ohkawa et al. was performed with slight changes. Lipid peroxidation was calculated using MDA as the standard. ${ }^{33}$ The tissue homogenate was prepared with Tris- $\mathrm{HCl}$ buffer was allowed to react with $20 \% \mathrm{w} / \mathrm{v}$ trichloroacetic acid (TCA) (freshly prepared). The reaction was carried out in cold environment. After centrifugation, the $2 \mathrm{ml}$ of supernatant was added to equal volume of freshly prepared TBA. The resulting solution was heated in a water bath for 60 mins. It was then cooled by keeping 
it on an ice bath for $15 \mathrm{~min}$. The formation of chromogen complex was measured at $532 \mathrm{~nm}^{33}$

\section{Determination of TNF- $\alpha$}

The sciatic nerves isolated from rats were homogenized in phosphate buffer saline $\mathrm{pH}$ 7.4. The mixture was centrifuged at $3800 \mathrm{rpm}$ and $100 \mu \mathrm{l}$ of the sample was used for TNF- $\alpha$ determination. TNF- $\alpha$ measurement was done as per the instructions given in kit.

\section{Effect of the extracts on body weight}

The body weights of the rats were recorded before the surgery and at the end of experimental period. The rats were also observed for changes in behavior, movement and other vital signs.

\section{RESULTS}

Antioxidant activity of the extracts was checked by DPPH method, Lipid Peroxidation method and Xanthine superoxide anion scavenging method. The results are depicted in Table 1.

\section{Pin Prick test}

Induction of TST surgery resulted in increase in paw withdrawal response for saline treated group as indicated in Figure 1. Treatment with all the licorice extract resulted in reduction in paw withdrawal duration of left hind paw after performing pin prick test. Effects of extracts were comparable to that of standard. The $\%$ Maximum Possible Effect (\%MPE) was calculated for all the extracts. (Table 2)

Figure 1: Effect of licorice extracts on mechanical hyperalgesia after TST surgery. Data is expressed as mean \pm standard error of mean (SEM), each group consisting of six $(n=6)$. Rats ${ }^{\mathrm{a}} p<0.05$ vs control group. ${ }^{\mathrm{b}} p<0.05$ vs sham control group.

\begin{tabular}{|c|c|c|c|c|}
\hline \multicolumn{5}{|c|}{ Table 1: Yield and properties of extracts. } \\
\hline Solvent & $\begin{array}{c}\text { Yield } \\
(\% w / w)\end{array}$ & Colour & Consistency & Solubility \\
\hline Methanol & 18.9 & $\begin{array}{c}\text { Mustard } \\
\text { brown }\end{array}$ & $\begin{array}{c}\text { Semi solid, } \\
\text { sticky }\end{array}$ & $\begin{array}{c}\text { Soluble in } \\
\text { methanol }\end{array}$ \\
\hline Ethanol & 20.6 & $\begin{array}{c}\text { Mustard } \\
\text { brown }\end{array}$ & $\begin{array}{c}\text { Semi solid, } \\
\text { sticky }\end{array}$ & $\begin{array}{c}\text { Soluble in } \\
\text { methanol }\end{array}$ \\
\hline $\begin{array}{c}\text { Hydroalcohol } \\
(50: 50)\end{array}$ & 37.55 & $\begin{array}{c}\text { Mustard } \\
\text { brown }\end{array}$ & $\begin{array}{c}\text { Semi solid, } \\
\text { sticky }\end{array}$ & $\begin{array}{c}\text { Soluble in } \\
\text { methanol }\end{array}$ \\
\hline $\begin{array}{c}\text { Ethyl acetate } \\
\text { brown }\end{array}$ & 10.95 & $\begin{array}{c}\text { Mustard } \\
\text { brown }\end{array}$ & $\begin{array}{c}\text { Free flowing } \\
\text { powder }\end{array}$ & $\begin{array}{c}\text { Soluble in } \\
\text { methanol }\end{array}$ \\
\hline $\begin{array}{c}\text { Distilled water } \\
\text { pown }\end{array}$ & 59.57 & $\begin{array}{c}\text { Mustard } \\
\text { brown }\end{array}$ & $\begin{array}{c}\text { Semi solid, } \\
\text { sticky }\end{array}$ & $\begin{array}{c}\text { Soluble in } \\
\text { methanol }\end{array}$ \\
\hline
\end{tabular}

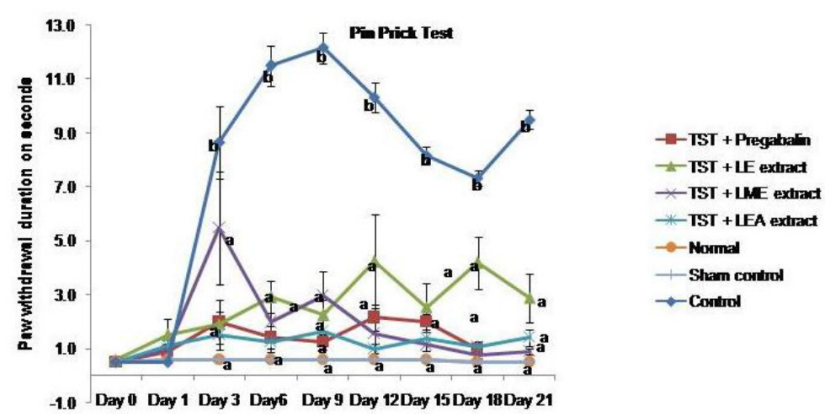

Figure 1: Effect of licorice extracts on mechanical hyperalgesia after TST surgery.

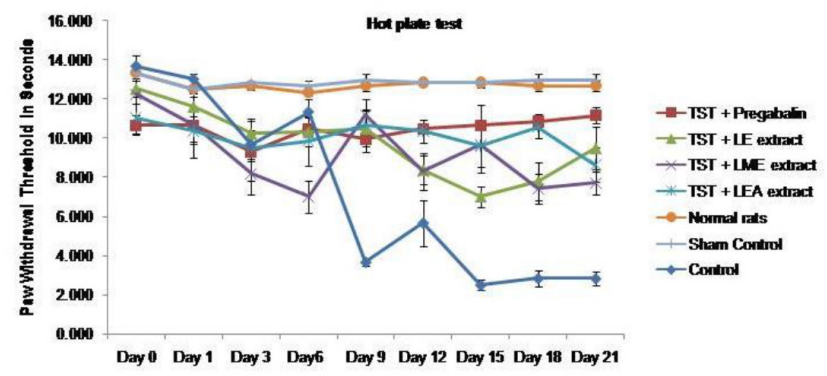

Figure 2: Effect of licorice extracts on Thermal hyperalgesia after TST surgery.

\begin{tabular}{|c|c|c|c|}
\multicolumn{4}{|c|}{ Table 2: Results of antioxidant activities of extracts } \\
by different methods. \\
$\begin{array}{c}\text { Solvent for } \\
\text { Extraction }\end{array}$ & $\begin{array}{c}\text { DPPH } \\
\text { method } \\
\left(\mathbf{I C}_{50}\right) \boldsymbol{\mu g} / \mathrm{ml}\end{array}$ & $\begin{array}{c}\text { Lipid } \\
\text { peroxidation } \\
\text { method }\left(\mathbf{I C}_{50}\right) \\
\boldsymbol{\mu g} / \mathrm{ml}\end{array}$ & $\begin{array}{c}\text { Xanthine } \\
\text { superoxide } \\
\text { method }\end{array}$ \\
\hline $\begin{array}{c}\text { Methanol } \\
\text { (LME Extract) }\end{array}$ & 71.08 & 882.91 & $\begin{array}{c}75.50 \% \\
\text { inhibition at } \\
600 \mathrm{ppm}\end{array}$ \\
\hline $\begin{array}{c}\text { Ethanol } \\
\text { (LE Extract) }\end{array}$ & 69.00 & 272.85 & $\begin{array}{c}55.91 \% \\
\text { inhibition at } \\
600 \mathrm{ppm}\end{array}$ \\
\hline $\begin{array}{l}\text { Ethyl acetate } \\
\text { (LEA Extract) }\end{array}$ & 38.59 & 218.34 & $\begin{array}{c}41.51 \% \text { at } \\
600 \mathrm{ppm}\end{array}$ \\
\hline
\end{tabular}

\section{Hot plate test}

TST surgery resulted in reduction in paw withdrawal threshold in saline treated group as depicted in Figure 2. Following treatment with licorice extracts, increase in TST induced paw withdrawal threshold was observed. The $\% \mathrm{MPE}$ was calculated for all the extracts. (Table 2) Figure 2: Effect of licorice extracts on Thermal hyperalgesia after TST surgery. Results are presented as mean \pm standard error of mean (SEM), each group consisting of six $(n=6)$ rats. ${ }^{a} p<0.05$ vs control group. ${ }^{b} p<0.05$ vs sham control group. 


\section{Acetone drop test}

Introduction of TST surgery resulted in increase in paw withdrawal duration as tested by acetone drop method as shown in Figure 3. Treatment with licorice extracts resulted in significant reduction in paw withdrawal duration of left hind limb of rat. Extracts showed decrease in paw withdrawal duration comparable to standard. The \%MPE was calculated for all the extracts. (Table 2) Figure 3: Effect of licorice extracts on Cold Allodynia after TST surgery. Results are presented as mean \pm standard error of mean (SEM), each group consisting of six $(n=6)$. Rats. ${ }^{\mathrm{a}} \not<<0.05$ vs control group. ${ }^{\mathrm{b}} \not<<0.05$ vs sham control group.

\section{Paint Brush Test}

There was an increase in paw withdrawal duration in left hind limb of rat, following TST surgery as shown in Figure 4. Treatment with licorice extracts resulted in significant decline in paw withdrawal duration of left hind limb of rat. Extracts showed decrease in paw withdrawal duration comparable to standard. The $\% \mathrm{MPE}$ was calculated for all the extracts. (Table 2)

Figure 4: Effect of licorice extracts on Mechanical dynamic allodynia after TST surgery. Results are presented as mean \pm standard error of mean (SEM), each group consisting of six $(n=6)$. Rats ${ }^{a} p<0.05$ vs control group. ${ }^{\mathrm{b}} p<0.05$ vs sham control group.
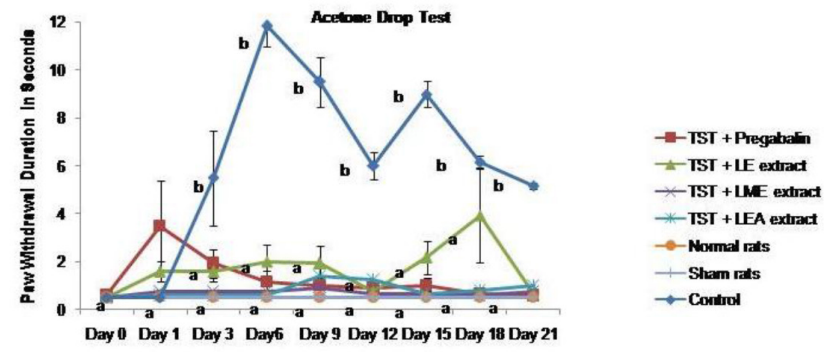

Figure 3: Effect of licorice extracts on Cold Allodynia after TST surgery.

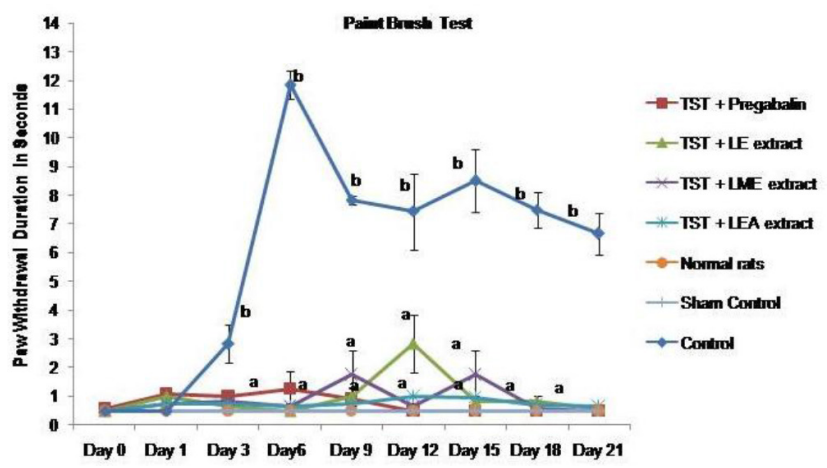

Figure 4: Effect of licorice extracts on Mechanical dynamic allodynia after TST surgery.

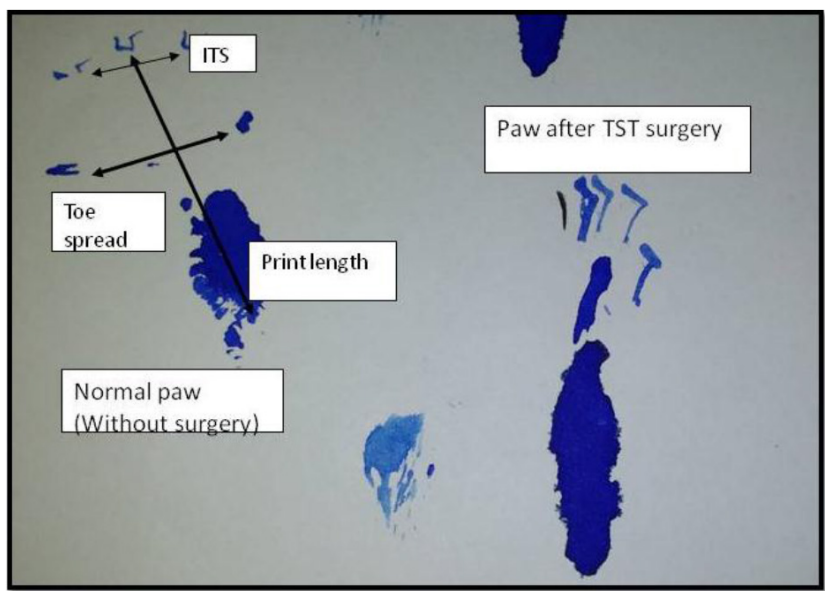

Figure 5: The footprint of rats, left indicates normal paw and footprint on right indicates damaged position of hind limb and digits after surgery.

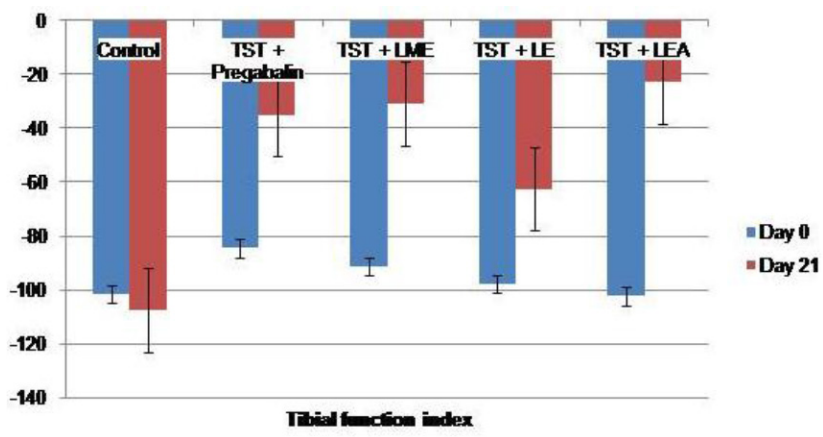

Figure 6: Effect of licorice extracts on Tibial Function Index after TST surgery.

Tibial function index: TST surgery causes deformities in positioning of limb and digits as shown in Figure 5. Deformities which are developed in hind limb result in negative value of tibial function index. Tibial function index near to the value of -100 , indicates complete loss of function. Treatment with licorice extracts resulted in improvement in tibial function index as indicated in Figure 6.

Figure 5: The footprint on left indicates normal paw and footprint on right indicates damaged position of hind limb and digits after surgery. The \%MPE was calculated for all the extracts.

Figure 6: Effect of licorice extracts on Tibial Function Index after TST surgery. Results are presented as mean \pm standard error of mean (SEM), each group consisting of six $(n=6)$ rats.

At the end of 21 days, animals were sacrificed and various biochemical parameters were estimated from tissue homogenates. The effects of licorice extracts on tissue biomarker changes are shown in Table 3. 


\begin{tabular}{l} 
Table 3: \% Maximum Possible Effect (MPE) of extracts \\
in behavioral tests in treated rats in comparison with \\
Control Group. \\
\hline Extract \\
\begin{tabular}{c|c|c|c|c|c|}
\hline $\begin{array}{c}\text { MPE\% } \\
\text { Pin Prick } \\
\text { Method }\end{array}$ & $\begin{array}{c}\text { MPE\% } \\
\text { Acetone } \\
\text { Drop } \\
\text { Method }\end{array}$ & $\begin{array}{c}\text { MPE\% } \\
\text { Hot Plate } \\
\text { Method }\end{array}$ & $\begin{array}{c}\text { MPE \% } \\
\text { Paint } \\
\text { Brush } \\
\text { Method }\end{array}$ & $\begin{array}{c}\% \text { MPE } \\
\text { TFI }\end{array}$ \\
\hline Methanol & $90.543^{\mathrm{b}}$ & $85.483^{\mathrm{a}, \mathrm{b}}$ & $40.054^{\mathrm{a}, \mathrm{b}}$ & 92.50 & 66.02 \\
\hline Ethanol & $69.666^{\mathrm{a}, \mathrm{b}}$ & $87.096^{\mathrm{a}, \mathrm{b}}$ & $54.767^{\mathrm{a}, \mathrm{b}}$ & $91.25^{\mathrm{a}, \mathrm{b}}$ & 35.95 \\
\hline $\begin{array}{c}\text { Ethyl } \\
\text { acetate }\end{array}$ & $85.052^{\mathrm{a}, \mathrm{b}}$ & $80.935^{\mathrm{a}, \mathrm{b}}$ & $47.753^{\mathrm{a}, \mathrm{b}}$ & $90.00^{\mathrm{a}, \mathrm{b}}$ & 77.56 \\
\hline Standard & $89.473^{\mathrm{b}}$ & $88.709^{\mathrm{b}}$ & $68.520^{\mathrm{b}}$ & 92.50 & 58.62 \\
\hline Sham & $94.736^{\mathrm{a}}$ & $90.322^{\mathrm{a}}$ & $78.205^{\mathrm{a}}$ & 92.50 & \\
\hline
\end{tabular}
\end{tabular}

For behavioral tests: Dunnet test a: $p<0.01$ compared to standard, $\mathrm{b}: p<0.01$ compared to sham, c: $p<0.05$ compared to standard.

\begin{tabular}{|c|c|c|c|c|c|c|c|}
\hline 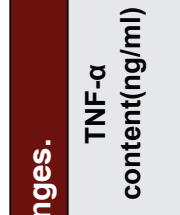 & 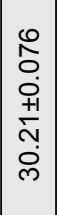 & 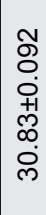 & 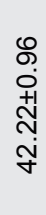 & \begin{tabular}{l}
0 \\
\hdashline \\
0 \\
+1 \\
0 \\
0 \\
0 \\
0
\end{tabular} & 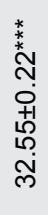 & 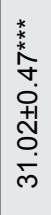 & 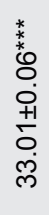 \\
\hline 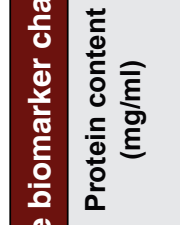 & 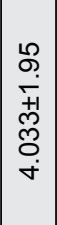 & $\begin{array}{l}10 \\
0 \\
0 \\
+1 \\
0 \\
0 \\
0 \\
0\end{array}$ & $\begin{array}{l}\stackrel{0}{0} \\
0 \\
+1 \\
1 \\
\infty \\
0 \\
0 \\
0\end{array}$ & $\begin{array}{l}\infty \\
0 \\
0 \\
+1 \\
0 \\
0 \\
0 \\
0\end{array}$ & 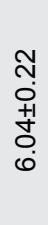 & 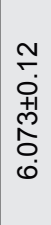 & 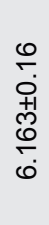 \\
\hline 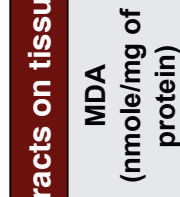 & 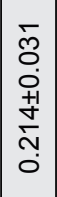 & $\begin{array}{l}\text { Jे } \\
0 \\
0 \\
+1 \\
+1 \\
\dot{+} \\
\vdots\end{array}$ & $\begin{array}{l}\text { o } \\
0 \\
0 \\
+1 \\
0 \\
\infty \\
0 \\
0\end{array}$ & 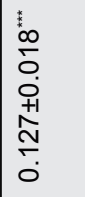 & $\begin{array}{l}1 \\
1 \\
0 \\
0 \\
0 \\
0 \\
0 \\
\\
0\end{array}$ & $\begin{array}{l}1 \\
\infty \\
0 \\
0 \\
0 \\
+1 \\
\infty \\
\stackrel{1}{0} \\
\vdots \\
0\end{array}$ & 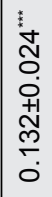 \\
\hline 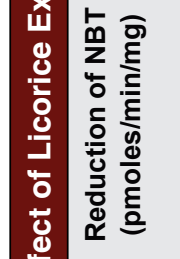 & 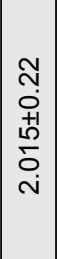 & $\begin{array}{l}0 \\
0 \\
0 \\
0 \\
+1 \\
0 \\
0 \\
0 \\
0 \\
\text { N. }\end{array}$ & \begin{tabular}{l}
$m$ \\
\multirow{+}{*}{} \\
+ \\
+1 \\
$\tilde{N}$ \\
$\infty$ \\
$\dot{\sigma}$
\end{tabular} & 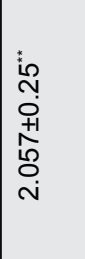 & 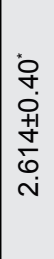 & 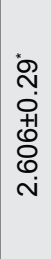 & 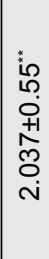 \\
\hline $\begin{array}{l}\text { o } \\
\frac{0}{3} \\
\frac{0}{j}\end{array}$ & $\mid \begin{array}{c}\overline{\widetilde{\sigma}} \\
\text { है } \\
\text { 之े }\end{array}$ & $\begin{array}{l}\frac{E}{\sigma} \\
\frac{\sigma}{\omega}\end{array}$ & 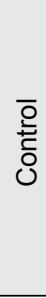 & 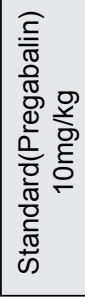 & 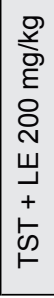 & 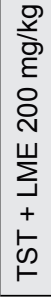 & 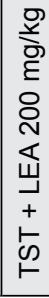 \\
\hline
\end{tabular}

Figure 7: Effect of extracts on histopathological changes. A Normal, B Control, C, Sham, D, Standard (TST + Pregabalin), E: (TST + LME), F: (TST + LE), G: (TST + LEA). TST resulted in significant histopathological changes in transverse sections of sciatic nerve. Dilation of myelin sheath was observed indicating damage to axons seen in control rats, indicated by black arrows. Treatment with licorice extracts have shown to preserve the myelin sheath.
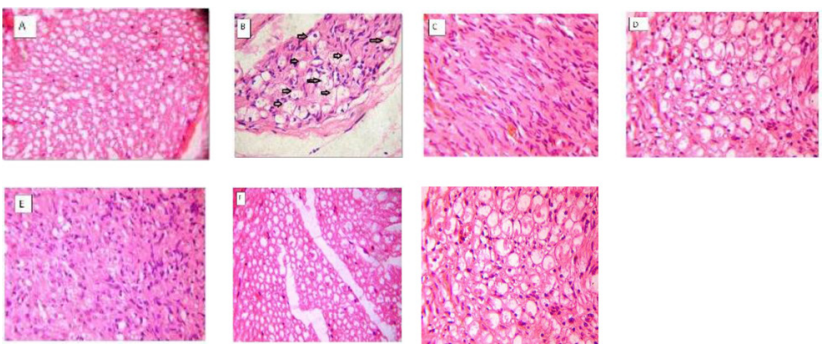

Figure 7: Histopathological studies of sciatic nerves of rats.A Normal, B Control, C, Sham, D, Standard (TST + Pregabalin), E: (TST + LME), F: (TST + LE), G: (TST + LEA). TST resulted in significant histopathological changes in transverse sections of sciatic nerve. Dilation of myelin sheath was observed indicating damage to axons seen in control rats, indicated by black arrows. Treatment with licorice extracts have shown to preserve the myelin sheath.

\section{Effect of the extracts on body weight}

Body weights of the rats were recorded before the surgery and at the end of experimental period. The body weights were found to be normal and the extracts were not shown produce any toxic effects with respect to weight and other vital signs of animals. The rats in the control group were observed to move and walk slowly in the cage, as a consequence of TST surgery. Treated rats showed improvement in walking which shown by improvement in tibial function index.

\section{DISCUSSION}

In the present study the extraction was carried out with different solvent and further total phenolic content and total flavonoid content of the extracts were determined. Phenolic compounds are considered to be most important group of antioxidants mainly because of their redox properties. It is reported that phenolic and flavonoid compounds act as antioxidant and they protect against oxidative stress and reduce oxidation of low density lipoproteins. ${ }^{34}$ Hence the extracts with higher phenolic and flavonoid content were tested for antioxidant activity.

DPPH scavenging method is one of the commonest methods for testing antioxidant activity. The extracts were allowed to react with DPPH stable radical and decrease in absorbance was considered as antioxidant capacity. The extracts found to have better free radical scavenging activity by DPPH method, were further tested for superoxide scavenging and lipid peroxidation scavenging activity. Production of ROS plays a major role in development of chronic neuropathic pain. Cellular stress or trauma causes excessive intracellular amounts of ROS, which can overcome the functions of homeostatic proteins and cause oxidative damage 
to the cell. Neurons are especially sensitive to ROS as they have greater energy demands for functioning as compared to glial and other cells in nervous system. In preclinical models of neuropathic pain, it was shown that symptoms of neuropathic pain were improved by systemic administration of antioxidants. ${ }^{35}$ Oxidative degeneration of lipids causes lipid peroxidation. In this process free radicals "steal" electrons from lipids in cell membranes, causing damage to cell. The secondary products of ROS, which are called as lipid peroxidation (LP) products, also contribute to pathophysiology of neuropathic pain. ${ }^{36}$ It was found that ethanol, methanol and ethyl acetate extracts of the Glycyrrbiza glabra possessed significant antioxidant activity against superoxides and LP products.

The neuropathic pain was induced by TST injury. The TST injury produced symptoms like thermal hyperalgesia, mechanical hyperalgesia, cold allodynia and mechanical dynamic allodynia. This surgical method is consistent and reproducible as compared to CCI. In CCI, the pain symptoms may vary as the tension on the ligatures may differ from one animal to the other. The Glycyrrbiza glabra extracts showed significant reduction in all the symptoms along with improving tibial function index. The treatment with extracts also showed decrease is various biochemical parameters like superoxide generation, TBAR content and TNF- $\alpha$ content. TST induced symptoms were observed day 3 onwards and symptoms were shown throughout the experimental period. Neuropathic pain encompasses two pathophysiological factors i.e. oxidative stress and inflammation. Under normal cellular homeostatic conditions, ROS is removed from cells by the action of enzymes such as superoxide dismutase (SOD), catalase or glutathione. Antioxidants vitamin $\mathrm{C}$ and $\mathrm{E}$ also contribute to scavenging of ROS. In pathological conditions, such as inflammation and pain, intracellular ROS level is increased due to augmented production or impaired removal of ROS. Increased ROS causes cell damage leading to death. Removal of excessive ROS is often important for restoring and maintaining homeostatic conditions. Studies have reported improvement in hyperalgesia symptoms after administration of antioxidants, in CCI model of neuropathic pain. ${ }^{37,38}$ It is reported that proinflammatory cytokines like tumor necrosis factor-alpha (TNF- $\alpha$ ) play major role in pathophysiology of neuropathic pain by producing axonal degeneration, demyelination. TNF- $\alpha$ is found to induce thermal hyperalgesia when injected into the endoneurium and it has also shown to evoke ectopic activity in isolated nerve fibers when applied topically. In a study conducted by Sommer et al. it was found that hyperalgesia after nerve injury can be reduced by neutralizing antibodies against TNF. ${ }^{39,40}$

In the present study; ethanol, methanol, ethyl acetate extracts of Glycyrrbiza glabra has shown beneficial effects in reducing various symptoms of TST induced neuropathic pain. The rota rod test was also carried out to test effect of neuropathic pain on the muscle grip, but it was found that neuropathic pain symptoms did not affect the grip.

As per the literature search, the methanol extracts of licorice are reported to have various phytoconstituents such as neoliquiritin, liquiritin, neoisoliquiritin, isoliquiritin, glycyrrhizin, isoliquiritigenin, licoisoflavone, licoflavone, glabrene, kumatakenin, licochalcone, glycyrol, glabridine, glabrone, licoflavonol, glycyrin, licoisoflavone $\mathrm{B}$, glabrol. ${ }^{41}$ The ethanol extracts are reported to have glycyrrhizic acid, glabridin, liquiritin, liquiritigenin. ${ }^{42}$ The ethyl acetate extracts are reported to have glycycoumarin, glisoflavone, glycyrol, licoisoflavanone, isowighteone, glycyrin, isoangustone, glyasperin D, glicophenone, licoricidin and 39-(g,gdimethylallyl)-kievitone. ${ }^{43}$ Glycyrrhizin (glycyrrhizic acid; glycyrrhizinate) is considered to be primary active ingredient of licorice root extract. It constitutes up to $10-25 \%$ of licorice root extract. ${ }^{44}$ Glycyrrhizin is metabolized to its active metabolite, aglycone $18 \beta$-glycyrrhetinic acid which is known to possess anti-inflammatory activity. ${ }^{42}$ The extracts were subjected to LC-MS analysis and they were found to contain presence of carbenexolone, a glycyerrhetenic acid derivate. The antinociceptive effect of the extract could be due to presence of glycyrrhetinic acid derivative along with other phytoconstituents present in extracts. The extracts were shown to inhibit superoxides and lipid peroxidation products in in vitro antioxidant studies and similar results were obtained the biochemical studies which were performed on the tissue homogenates; hence confirming in vitro and in vivo correlation. The extracts were shown to attenuate TNF- $\alpha$ level in tissue homogenates. Histopathological studies of the nerves of rats of all groups were carried out. It was observed that, in the rats belonging to control group there was dilatation of myelin sheath in $50 \%$ of the nerve indicating axonal damage, whereas in treated rats, the myelin sheath was preserved.

Liquritin has been shown to improve symptoms of neuropathy in CCI induced rats. ${ }^{45}$ Ethyl acetate is one of the common solvents used to extract nonglycosylated flavonoids, coumaric and phenolic-carboxylic acids and coumarins. Flavonoids and coumarins are well known for their free radical scavenging activities. The same has been indicated by $\mathrm{IC}_{50}$ values obtained in 
various antioxidant methods. Hence, it can be postulated that anti-inflammatory and antioxidant activity possessed by extracts could be contributing to reduction is symptoms of neuropathic pain in rats.

\section{CONCLUSION}

Glycyrriza glabra extracts effectively reduced symptoms of TST induced neuropathic pain in rats. This effect can be attributed to antioxidant, anti-inflammatory and neuroprotective actions of Glycyrriza glabra. Thus, extracts of Glycyrriza glabra can be promising leads used in treatment of neuropathic pain.

\section{ACKNOWLEDGEMENT}

Authors are thankful to SUUTI grant from SNDT University for providing financial assistance for purchase of TNF- $\alpha$ kit.

\section{CONFLICT OF INTEREST}

Authors declare that there are no conflicts of interest.

\section{ABBREVIATIONS}

NP: Neuropathic pain; TST: Tibial and Sural Transection; MDA: Malondialdehyde; ROS: Reactive oxygen species; CCI: Chronic Constriction Injury; NBT: Nitro Blue Tetrazolium; DPPH: 2, 2'-diphenyl-1- picrylhydrazyl; BBE: Bovine Brain Extract; TPC: Total Phenolic Content; PBS: Phosphate Buffer Saline; TBA: Thiobarbituric Acid; LE: Licorice Ethanol Extract; LME: Licorice Methanol Extract; LEA: Licorice Ethyl acetate Extract; TCA: Trichloro Acetic acid; \%MPE: \% Maximum Possible Effect; LP: Lipid Peroxidation; SOD: Superoxide Dismutase.

\section{REFERENCES}

1. International Association for the Study of Pain: Pain Definitions. Pain Repots. 2018;e643. https://www.iasp-pain.org/Taxonomy, retrieved on $17^{\text {th }}$ January 2018. Last Updated: December 14, 2017

2. Smith HS, Torrance N. Epidemiology of Neuropathic pain and its impact on life. Curr Pain Headache Rep. 2012;16(3):191-8.

3. Koltzenburg M, Scadding J. Neuropathic pain. Current Opinion in Neurology. 2001;14(5):641-7.

4. Colombo B, Annovazzi POL, Comi G. Medications for neuropathic pain: Current trends. Neurology Science. 2006;27(2):S183-9.

5. Haanpa M, Treede RD. Epidemiology and impact of neuropathic pain. Pain Clinical Updates. 2010;18(7):1-7.

6. Lee FH, Raja SN. Complementary and alternative medicine in chronic pain. Pain. 2011;152(1):28-30.

7. Dowdall T, Robinson I, Meert TF. Comparison of five different rat models of peripheral nerve injury. Pharmacology, Biochemistry and Behavior. 2005;80(1):93-108.
8. Lee BH, Won R, Baik EJ, Lee SH, Moon CH. An animal model of neuropathic pain employing injury to the sciatic nerve branches. Neuroreport. 2000;11(4):657-61.

9. Woolf CJ, Mannion RJ. Neuropathic pain: Etiology, symptoms, mechanisms and management. Lancet Pain. 1999;353(9168):1959-64.

10. Nickel FT, Seifert F, Lanz S, Maihöfner C. Mechanism of neuropathic pain. European Psychopharmacology. 2012;22(2):81-91.

11. Tal M. A novel antioxidant alleviates heat hyperalgesia in rats with an experimental painful peripheral neuropathy. Neur Report. 1996;7(8):1382-4.

12. Khalil Z, Liu T, Helme RD. Free radicals contribute to the reduction in peripheral vascular responses and the maintenance of thermal hyperalgesia in rats with chronic constriction injury. Pain. 1999;79(1):31-7.

13. Sharma V, Adarwal RC. Glycyrrhiza glabra: A Plant for the Future. Mintage Journal of Pharmaceutical and Medical Sciences. 2013;15-20. (ISSN: 2320-3315)

14. Laxmi T, Geetha RVI. Glycyrrhiza glabra Linn Commonly Known As Licorice: A Therapeutic Review. Int J Pharm Pharm Sci. 2011;3(4):20-5.

15. Muthuraman A, Singh N. Attenuating effect of Acoruscalamus extract in chronic constriction injury induced neuropathic pain in rats: An evidence of anti-oxidative, anti-inflammatory, neuroprotective and calcium inhibitory effects. BMC Complementary and Alternative Medicine. 2011;11(24):1-14.

16. Kaur G, Jaggi AS, Singh N. Exploring the potential effect of Ocimum sanctum in vincristine induced neuropathic pain in rats. Journal of Brachial Plexus and Peripheral Never Injury. 2010;5(3):1-9.

17. Jeong HG, et al. Hepatoprotective effects of $18 \beta$-glycyrrhetinic acid on carbon tetrachloride-induced liver injury: Inhibition of cytochrome p450 2e1 expression. Pharmacological Research. 2002;46(3):221-7.

18. Andrew LW. Determination of Total Phenolics. Current Protocols in Food Analytical Chemistry. 2002;6(1):1.

19. Atanassova M, et al. Total Phenolic and Total Flavonoid Contents, Antioxidant Capacity and Biological Contaminants in Medicinal Herbs. Journal of the University of Chemical Technology and Metallurgy. 2011;46(1):81-8.

20. Halliwell B. Antioxidant characterization: Methodology and mechanism. Biochem Pharmacol. 1995;49(10):1341-8.

21. Ostrea EM, et al. Red Cell Membrane Lipid Peroxidation and Hemolysis Secondary to Phototherapy. Acta Paediatrica. 1985;74(3):378-81.

22. Sun YI, Oberley LW. A simple method for clinical assay of superoxide dismutase. Clin Chem. 1988;34(3):497-500.

23. Jain V, Jaggi AS, Singh N. Ameliorative potential of rosiglitazone in tibia and surely nerve transection-induced painful neuropathy in rats. Pharmacological Research. 2009;59(6):385-92.

24. Baron R, BinderA, Wasner G. Neuropathic pain: Diagnosis, pathophysiological mechanisms and treatment. Lancet Neurology. 2010;9(8):807-19.

25. Eddy NB, Touchberry CF, Lieberman JE. Synthetic analgesics: I. J Pharmacol Exp Ther. 1950;98(2):121-37.

26. Erichsen HK, Blackburn-Munro G. Pharmacological characterization of the spared nerve injury model of neuropathic pain. Pain. 2002;98(1-2):151-61.

27. Choi Y, Yoon YW, Na HS, Kim SH, Chung JM. Behavioural signs of ongoing pain and cold allodia in a rat model of neuropathic pain. Pain. 1994;59(3):369-76.

28. Ochoa JL, Yarnitsky D. Mechanical Hyperalgesias in Neuropahc Pain Patients: Dynamic and Static Subtypes. Ann Neurol. 1993;33(5):465-72.

29. Jain V, Pareek A, Bhardwaj YR, Singh N. Attenuating effect of standardized fruit extract of Punica granatum $\mathrm{L}$ in rat model of tibial and sural nerve transection induced neuropathic pain. BMC Complementary and Alternative Medicine. 2013;13(1):274.

30. Reis FA, Belchior ACG, et al. Effect of gallium-aluminum-arsenide laser therapy $(660 \mathrm{Nm})$ on recovery of the sciatic nerve in rats following neurotmesis lesion and epineural anastomosis: Functional analysis. Revista Brasileira De Fisioterapia. 2008;12(3):215-21.

31. Medinaceli L, Freed WJ, Wyatt RJ. An Index of the Functional Condition of Rat Sciatic Nerve Based on Measurements Made from Walking Tracks. Experimental Neurology. 1982;77(3):634-43

32. Wang HD, Pagano PJ, Du Y, Cayatte A, et al. Superoxide Anion from the Adventitia of the Rat Thoracic Aorta Inactivates Nitric Oxide. Circulation Research. 1998;82(7):810-8.

33. Ohkawa H, Ohishi N, Yagi K. Assay for Lipid Peroxides in Animal Tissues by Thiobarbituric Acid Reaction. Analytical Biochemistry. 1979;95(2):351-8. 
34. Huyut Z, Beydemir S, Gülçin I. Antioxidant and Antiradical Properties of Selected Flavonoids and Phenolic Compounds. Biochemistry Research International. 2017;1-10.

35. Hassler SN, Johnson KM, Hulsebosch CE. Reactive oxygen species and lipid peroxidation inhibitors reduce mechanical sensitivity in a chronic neuropathic pain model of spinal cord injury in rats. J Neurochem. 2014;131(4):413-7.

36. Naik AK, Tandan SK, Dudhgaonkar SP, Jadhav SH, Kataria M, Prakash VR, et al. Role of oxidative stress in pathophysiology of peripheral neuropathy and modulation by $\mathrm{N}$-acetyl-L-cysteine in rats. European Journal of Pain. 2006;10(7):573-9.

37. Gwak et al. Reactive oxygen species contribute to neuropathic pain and locomotor dysfunction via activation of Cam KII in remote segments following spinal cord contusion injury in rats. Pain. 2013;154(9):1699-708.

38. Khalil Z, Liu T, Helme R. Free radicals contribute to the reduction in peripheral vascularresponses and the maintenance of thermal hyperalgesia in rats with chronic constriction injury. Pain. 1999;79(1):31-7.

39. Leung L, Cahill C. TNF- $\alpha$ and neuropathic pain: A review. Journal of Neuroinflammation. 2010;7(1):27.

40. Sommer C, Schmidt C, George A. Hyperalgesia in Experimental Neuropathy Is Dependent on the TNF Receptor. Exp Neurol. 1998;151(1):138-42.
41. Hiraga $\mathrm{Y}$, Endo $\mathrm{H}$, Takahashi $\mathrm{K}$, Shibata S. High-performance liquid chromatographic analysis of licorice extracts. Journal of Chromatography. 1984;292(2):451-3.

42. Cao LJ, Hou ZY, Li HD, Zhang BK, Fang PF, Xiang DX, et al. The Ethanol Extract of Licorice Protects against Triptolide-Induced Oxidative Stress through Activation of $\mathrm{Nrf}_{2}$. Evidence-Based Complementary and Alternative Medicine. 2017. Article ID 2752389: 12 pages. https://doi. org/10.1155/2017/2752389

43. Denisova SB, Galkin EG, Murinov YI. Isolation and GC-MS determination of flavonoids from Glycyrrhiza glabra root. Chemistry of Natural Compounds. 2006;42(3):285-9.

44. Kaur R, Kaur H, Dhindsa AS. Glycyrrhiza glabra: A phytopharmacological review. IJPSR. 2013;4(7):2470-7.

45. Kalaiarasi P, Pugalendi KV. Protective effect of 18b-glycyrrhetinic acid on lipid peroxidation and antioxidant enzymes in experimental diabetes. J Pharm Res. 2011;4(1):107-11.

46. Zhang MT, Wang B, Jia YN Liu N, Ma PS, Gong SS, et al. Neuroprotective effect of liquiritin against neuropathic pain induced by chronic constriction injury of the sciatic nerve in mice. Biomed Pharmacother. 2017;95:186-98.

\section{PICTORIAL ABSTRACT}

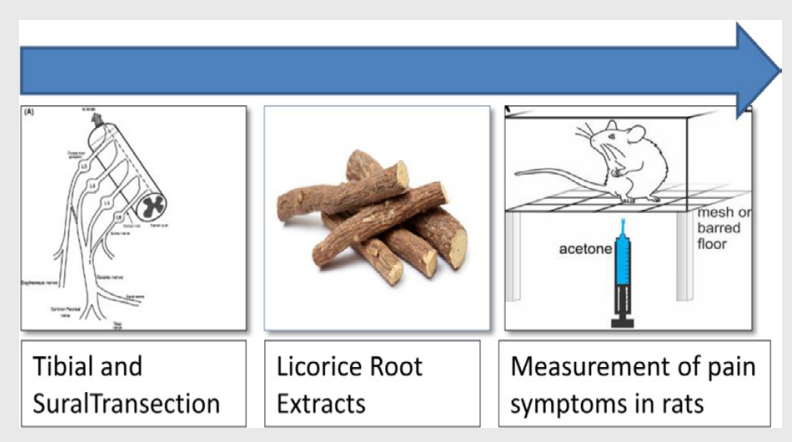

\section{SUMMARY}

Licorice extracts were shown to reduce symptoms of neuropathic pain in preclinical tibial and sural transection model.

Cite this article: Joshi TA, Tatke P. Neuroprotective Effects of Licorice Extracts in Tibial and Sural Transection Induced Neuropathic Pain in Rats. Indian J of Pharmaceutical Education and Research. 2020;54(2s):s285-s294. 